Article

\title{
History of Heart Failure in Patients Hospitalized Due to COVID-19: Relevant Factor of In-Hospital Complications and All-Cause Mortality up to Six Months
}

\author{
Mateusz Sokolski 1,2,*(D), Konrad Reszka ${ }^{2}$ (D) , Tomasz Suchocki ${ }^{3}$ (D), Barbara Adamik ${ }^{4}$, Adrian Doroszko ${ }^{5}$, \\ Jarosław Drobnik ${ }^{6}$, Joanna Gorka-Dynysiewicz ${ }^{7}$ (D), Maria Jedrzejczyk ${ }^{8}{ }^{(D)}$, Krzysztof Kaliszewski ${ }^{9}$, \\ Katarzyna Kilis-Pstrusinska ${ }^{10}$, Bogusława Konopska ${ }^{11}$ (D), Agnieszka Kopec ${ }^{12}$, Anna Larysz 2,13, Weronika Lis ${ }^{2}$, \\ Agnieszka Matera-Witkiewicz ${ }^{14}$, Lilla Pawlik-Sobecka ${ }^{15}$, Marta Rosiek-Biegus ${ }^{12}$, Justyna M. Sokolska ${ }^{1,2}$, \\ Janusz Sokolowski ${ }^{16}$, Anna Zapolska-Tomasiewicz ${ }^{1}$, Marcin Protasiewicz ${ }^{1,2}$, Katarzyna Madziarska ${ }^{17,+}$ \\ and Ewa A. Jankowska 1,2, + $\mathbb{D}$
}

check for updates

Citation: Sokolski, M.; Reszka, K.; Suchocki, T.; Adamik, B.; Doroszko, A.; Drobnik, J.; Gorka-Dynysiewicz, J.; Jedrzejczyk, M.; Kaliszewski, K.; Kilis-Pstrusinska, K.; et al. History of Heart Failure in Patients Hospitalized Due to COVID-19: Relevant Factor of In-Hospital Complications and All-Cause Mortality up to Six Months. J. Clin. Med. 2022, 11, 241. https://doi.org/ $10.3390 /$ jcm 11010241

Academic Editor: Juan F. Delgado Jiménez

Received: 28 November 2021 Accepted: 30 December 2021 Published: 3 January 2022

Publisher's Note: MDPI stays neutral with regard to jurisdictional claims in published maps and institutional affiliations.

Copyright: (C) 2022 by the authors. Licensee MDPI, Basel, Switzerland. This article is an open access article distributed under the terms and conditions of the Creative Commons Attribution (CC BY) license (https:// creativecommons.org/licenses/by/ $4.0 /)$.
1 Institute of Heart Diseases, Wroclaw Medical University, Borowska 213, 50-556 Wroclaw, Poland; justyna.sokolska@umw.edu.pl (J.M.S.); anna.zapolska@umw.edu.pl (A.Z.-T.); marcin.protasiewicz@umw.edu.pl (M.P.); ewa.jankowska@umw.edu.pl (E.A.J.)

2 Institute of Heart Diseases, University Hospital, 50-556 Wroclaw, Poland; koreszka@usk.wroc.pl (K.R.); anna.larysz@umw.edu.pl (A.L.); wlis@usk.wroc.pl (W.L.)

3 Biostatistics Group, Department of Genetics, Wroclaw University of Environmental and Life Sciences, 51-631 Wroclaw, Poland; tomasz.suchocki@gmail.com

4 Department of Anesthesiology and Intensive Therapy, Wroclaw Medical University, 50-367 Wroclaw, Poland; barbara.adamik@umw.edu.pl

5 Clinical Department of Internal and Occupational Diseases, Hypertension and Clinical Oncology, Wroclaw Medical University, 50-556 Wroclaw, Poland; adrian.doroszko@umw.edu.pl

6 Gerontology Unit, Public Health Department, Wroclaw Medical University, 51-618 Wroclaw, Poland; jaroslaw.drobnik@umw.edu.pl

7 Department of Pharmaceutical Biochemistry, Division of Pharmaceutical Biochemistry, Wroclaw Medical University, 50-556 Wroclaw, Poland; joanna.gorka-dynysiewicz@umw.edu.pl

8 Department of Nursing and Obstetrics, Division of Internal Medicine Nursing, Wroclaw Medical University, 51-618 Wroclaw, Poland; maria.jedrzejczyk@umw.edu.pl

9 Clinical Department of General, Minimally Invasive and Endocrine Surgery, Wroclaw Medical University, 50-556 Wroclaw, Poland; krzysztof.kaliszewski@umw.edu.pl

10 Clinical Department of Paediatric Nephrology, Wroclaw Medical University, 50-556 Wroclaw, Poland; katarzyna.kilis-pstrusinska@umw.edu.pl

11 Department of Pharmaceutical Biochemistry, Wroclaw Medical University, 50-556 Wroclaw, Poland; boguslawa.konopska@umed.wroc.pl

12 Clinical Department of Internal Medicine, Pneumology and Allergology, Wroclaw Medical University, 50-369 Wroclaw, Poland; agnieszka.kopec@umw.edu.pl (A.K.); marta.rosiek-biegus@umw.edu.pl (M.R.-B.)

13 Clinical Department of Heart Transplantation and Mechanical Circulatory Support, Institute of Heart Disease, Wroclaw Medical University, 50-556 Wroclaw, Poland

14 Wroclaw Medical University Biobank, Wroclaw Medical University, 50-556 Wroclaw, Poland; agnieszka.matera-witkiewicz@umw.edu.pl

15 Division of Basic Sciences, Faculty of Health Sciences, Wroclaw Medical University, 50-368 Wroclaw, Poland; lilla.pawlik-sobecka@umw.edu.pl

16 Clinical Department of Emergency Medicine, Wroclaw Medical University, 50-556 Wroclaw, Poland; janusz.sokolowski@umw.edu.pl

17 Department of Nephrology and Transplantation Medicine, Wroclaw Medical University, 50-556 Wroclaw, Poland; katarzyna.madziarska@umw.edu.pl

* Correspondence: mateusz.sokolski@umw.edu.pl; Tel.: +48-717331112

+ These authors contributed equally to this work.

Abstract: Background: Patients with heart failure (HF) are at high risk of unfavorable courses of COVID-19. The aim of this study was to evaluate characteristics and outcomes of COVID-19 patients with HF. Methods: Data of patients hospitalized in a tertiary hospital in Poland between March 2020 and May 2021 with laboratory-confirmed COVID-19 were analyzed. The study population was divided into a HF group (patients with a history of HF) and a non-HF group. Results: Out of 2184 patients ( $65 \pm 13$ years old, $50 \%$ male), $12 \%$ had a history of HF. Patients from the HF group were 
older, more often males, had more comorbidities, more often dyspnea, pulmonary and peripheral congestion, inflammation, and end-organ damage biomarkers. HF patients had longer and more complicated hospital stay, with more frequent acute HF development as compared with non-HF. They had significantly higher mortality assessed in hospital (35\% vs. $12 \%$ ) at three (53\% vs. $22 \%$ ) and six months (72\% vs. $47 \%$ ). Of 76 (4\%) patients who developed acute $\mathrm{HF}, 71 \%$ died during hospitalization, $79 \%$ at three, and $87 \%$ at six months. Conclusions: The history of HF identifies patients with COVID-19 who are at high risk of in-hospital complications and mortality up to six months of follow-up.

Keywords: COVID-19; SARS-CoV-2; heart failure; morbidity; mortality; long-term outcome

\section{Introduction}

Heart failure (HF) is one of the most common causes of death worldwide [1]. Approximately 64.3 million people currently live with $\mathrm{HF}$, and the prevalence is estimated at $1-2 \%$ of the adult population in developed countries [2,3]. According to the studies of the HF Working Group of the Polish Cardiac Society based on reports of the National Health Fund, about 1.2 million people suffer from HF and 140 thousand die every year in Poland [4]. Despite the decline in cardiovascular mortality in the last decades, the prognosis in HF remains still poor. Approximately $24 \%$ of patients die within one year of HF diagnosis [5]. In-hospital mortality during HF hospitalizations is also unacceptably high and varies from 12 to $19 \%$, depending on consecutive years, gender, and age groups [6].

The outbreak of coronavirus disease 2019 (COVID-19) pandemic caused by severe acute respiratory syndrome coronavirus 2 (SARS-CoV-2) became a global health crisis that resulted in almost 4.9 million deaths worldwide until October 2021, while more than 241 million people were infected, and these numbers are still growing [7]. The clinical course of the SARS-CoV-2 infection range from asymptomatic to critical presentation [8]. While the severe and critical represented 21\% of COVID-19 patients, the in-hospital infection fatality rates vary from $11 \%$ to $31 \%$ [8-10]. Three and six months all-cause mortality in patients hospitalized with COVID-19 was estimated at $28 \%$ and $30 \%$, respectively [11]. However, the overall mortality rate in the population is lower ranging $0.3-5 \%[8,12,13]$.

Numerous risk factors for severe disease and death have been described since the beginning of the pandemic, including age, gender, lifestyle, laboratory indications, complications, and comorbidities [14]. Older age is associated with the risk of unfavorable course of the disease. In the United States, deaths of patients aged 50 years and older account for $>94 \%$ of the total deaths due to SARS-CoV-2 infection $[15,16]$. While gender does not affect the risk of infection, in males with COVID-19, poorer outcomes and more deaths are observed, independent of age [17].

Patients with HF are at especially high risk of morbidity and mortality from SARSCoV-2 infection [18]. COVID-19 is associated with an immune response leading to systemic inflammation [19]. Increased metabolic demands related to severe inflammatory reaction result in cardiac injury and myocardial functional disorders [18]. Moreover, the volume overload secondary to acute kidney injury (observed in up to $30 \%$ of patients with COVID-19), symptomatic treatment, fluid administration can aggravate chronic HF as well $[13,19,20]$. As a result, COVID-19 can exacerbate a pre-existing HF or even cause it de novo [2].

Interestingly, the COVID-19 pandemic has significantly decreased the number of overall hospital admissions for HF [21-23]. While patients with HF less frequently selfreferred to the hospital, the number of HF patients brought by an ambulance increased [21]. Moreover, during the pandemic, HF patients were less frequently admitted in New York Heart Association (NYHA) Class II compared with the pre-pandemic period [22]. 
The multicenter, international retrospective cohort study showed that COVID-19 patients with HF are at increased risk for in-hospital death and in-hospital worsening of HF or acute HF de novo, which further increases in-hospital mortality [24].

Despite intensive investigation of cardiovascular diseases in COVID-19, still, little is known about the long-term outcomes of HF patients hospitalized with COVID-19. The aim of this study was to evaluate clinical characteristics, in-hospital course, and posthospital outcomes of this group.

\section{Materials and Methods}

\subsection{Study Population}

The single-center retrospective cohort study included consecutive adult patients hospitalized in the University Hospital, Wroclaw, Poland between March 2020 and May 2021 with laboratory-confirmed COVID-19 defined as a positive result by polymerase chain reaction testing of a nasopharyngeal sample or a positive blood antigen test. The study population was divided into two subgroups:

(a) HF group: Patients with a history of $\mathrm{HF}$, diagnosed before hospitalization, ac-cording to the European Association of Cardiology (ESC) guidelines [25,26],

(b) Non-HF group: Patients without previous diagnosis of HF.

\subsection{End-Points of the Study and Clinical Follow-Up}

The primary endpoint was all-cause mortality at three months.

The secondary endpoints were:

(a) in-hospital mortality,

(b) duration of hospitalization,

(c) admission to intensive care unit (ICU),

(d) the incidence of complications during hospitalization: shock, myocardial infarc-tion (diagnosed according to Fourth Universal Definition of Myocardial Infarction [27]), thromboembolic disease, stroke, acute HF,

(e) all-cause mortality at six months.

\subsection{Study Procedures}

The demographic data, the information about medical history, previous medication, signs, symptoms at admission, laboratory tests, in-hospital clinical course were extracted from electronic medical records.

The following laboratory measurements were evaluated on admission: hematology: hemoglobin, leukocytes, lymphocytes, neutrophils; serum electrolytes: sodium, potassium; markers of infection: C-reactive protein (CRP), procalcitonin, interleukin-6 (IL-6), ferritin; renal and liver function tests: estimated glomerular filtration rate (eGFR), blood urea nitrogen (urea), uric acid, bilirubin, gamma-glutamyl transferase (GGTP), albumin; cardiac troponin I; plasma N-Terminal Pro-B-Type Natriuretic Peptide (NT-proBNP).

Surviving patients were followed by telephone contact after three and six months. Information was obtained directly from patients, their relatives, or from the hospital system. Government General Registry Office data regarding death were used to complete follow-up.

\subsection{Statistical Analysis}

The categorical variables were expressed as a number of patients in a given categories (with a percentage). The intergroup differences in categorical variables were tested using the $\chi^{2}$ test. Continuous variables with a normal distribution were expressed as a mean $(\bar{x})$ (with standard deviation (SD)). Continuous variables with a skewed distribution were described by median $(\mathrm{m})$ (with an interquartile range (IQR)). These variables were $\mathrm{ln}$ transformed in order to normalize their distribution, and ln-transformed values were used for further statistical analyses. The intergroup differences for continuous variables were tested using Student's $t$ test. The associations between the occurrence of acute HF and 
clinical and laboratory variables were tested using stepwise forward selection based on the Akaike criterion. In the results, we got the model with three independent variables (age, history of HF, and history of myocardial infarction). The associations between the clinical and laboratory variables and survival during 90 days in patients with COVID19 were established using Cox proportional hazard regression model (both univariable and multivariable models). Variables that were statistically significant in the univariable model were included in the multivariable model. To estimate the effect of history of HF on in-hospital, 3-months, and 6-months mortality, Kaplan-Meier curves were constructed. Differences in survival rates were tested with the log-rank test. The $p$-value $<0.05$ was considered statistically significant. Statistical analyses were performed using the R software version 3.5.1 (R Foundation for Statistical Computing, Vienna, Austria) and STATISTICA 13 data analysis software system (StatSoft, Inc., Tulsa, OK, USA).

\section{Results}

3.1. Characteristic of Study Population and Difference between Patients with a History of HF and without HF

The study included 2184 patients with a mean age of $65 \pm 13$ years, $1082(50 \%)$ were male. There were 255 (12\%) patients with a history of HF. Patients from the HF group were older, more often males, and had more comorbidities than patients without a history of HF. Before hospitalization, HF patients were more frequently treated with recommended HF medication, as well as calcium channel blockers, alpha-adrenergic blockers, statins, antiplatelet drugs, anticoagulants, antidiabetics, inhaled $\beta 2$-agonists, anticholinergic drugs, and hemodialysis (see Table 1).

Table 1. Baseline demographics, clinical characteristics, comorbidities, and related treatment in the studied cohorts.

\begin{tabular}{|c|c|c|c|c|}
\hline Variables, Units & $\begin{array}{l}\text { HF Group } \\
n=255\end{array}$ & $\begin{array}{c}\text { Non-HF Group } \\
n=1929\end{array}$ & $p$ & $\begin{array}{l}\text { Available Data, } \\
n(\%)\end{array}$ \\
\hline \multicolumn{5}{|c|}{ Demographics } \\
\hline Age, years & $75 \pm 12$ & $58 \pm 19$ & $<0.001$ & $2184(100)$ \\
\hline Age $\geq 70$ years, $n(\%)$ & $169(66)$ & $571(30)$ & $<0.001$ & $2184(100)$ \\
\hline Male gender, $n(\%)$ & $144(57)$ & $938(49)$ & 0.022 & $2184(100)$ \\
\hline Body mass index, $\mathrm{kg} / \mathrm{m}^{2}$ & $28 \pm 6$ & $28 \pm 5$ & 0.950 & $554(25)$ \\
\hline \multicolumn{5}{|c|}{ Co-Morbidities } \\
\hline Hypertension, $n(\%)$ & $215(84)$ & $807(42)$ & $<0.001$ & $2184(100)$ \\
\hline Diabetes mellitus, $n(\%)$ & $122(48)$ & $394(20)$ & $<0.001$ & $2182(100)$ \\
\hline Atrial fibrillation/flutter, $n(\%)$ & $134(53)$ & $156(8)$ & $<0.001$ & $2184(100)$ \\
\hline Previous coronary revascularization, $n(\%)$ & $93(37)$ & $61(3)$ & $<0.001$ & $2184(100)$ \\
\hline Previous myocardial infarction, $n(\%)$ & $92(36)$ & $99(5)$ & $<0.001$ & $2184(100)$ \\
\hline $\begin{array}{l}\text { Significant valvular heart disease or previous valve heart } \\
\text { surgery, } n(\%)\end{array}$ & $64(25)$ & $32(2)$ & $<0.001$ & $2184(100)$ \\
\hline Peripheral artery disease, $n(\%)$ & $43(17)$ & $57(3)$ & $<0.001$ & $2184(100)$ \\
\hline Previous stroke/transient ischemic attack, $n(\%)$ & $53(21)$ & $111(6)$ & $<0.001$ & $2184(100)$ \\
\hline Chronic kidney disease or/and hemodialysis, $n(\%)$ & $92(36)$ & $139(7)$ & $<0.001$ & $2184(100)$ \\
\hline Asthma, $n(\%)$ & $10(4)$ & $75(4)$ & 0.979 & $2184(100)$ \\
\hline Chronic obstructive pulmonary disease, $n(\%)$ & $29(11)$ & $46(2)$ & $<0.001$ & $2184(100)$ \\
\hline Cigarette smoking (previous or current), $n(\%)$ & $55(22)$ & $138(7)$ & $<0.001$ & $2180(100)$ \\
\hline
\end{tabular}


Table 1. Cont.

\begin{tabular}{|c|c|c|c|c|}
\hline Variables, Units & $\begin{array}{l}\text { HF Group } \\
n=255\end{array}$ & $\begin{array}{c}\text { Non-HF Group } \\
n=1929\end{array}$ & $p$ & $\begin{array}{c}\text { Available Data, } \\
n(\%)\end{array}$ \\
\hline Sleep apnea syndrome, $n(\%)$ & $7(3)$ & $9(1)$ & $<0.001$ & $2184(100)$ \\
\hline Chronic liver disease, $n(\%)$ & $15(6)$ & $59(3)$ & 0.031 & $2183(100)$ \\
\hline Thyroid disease, $n(\%)$ & $33(13) / 7(3)$ & $175(9) / 14(1)$ & 0.001 & $2184(100)$ \\
\hline Dementia, $n(\%)$ & $50(20)$ & $82(4.3)$ & $<0.001$ & $2184(100)$ \\
\hline Malignancy, $n(\%)$ & $29(11)$ & $156(8)$ & 0.099 & $2184(100)$ \\
\hline Transplant recipients, $n(\%)$ & $7(3)$ & $26(1)$ & 0.148 & $2184(100)$ \\
\hline \multicolumn{5}{|c|}{ Treatment Applied before Hospitalization } \\
\hline Angiotensin-converting enzyme inhibitors, $n(\%)$ & $104(41)$ & $248(13)$ & $<0.001$ & $2184(100)$ \\
\hline Angiotensin receptor blockers, $n(\%)$ & $24(9)$ & $120(6)$ & 0.073 & $2184(100)$ \\
\hline Mineralocorticoid receptor antagonists, $n(\%)$ & $56(22)$ & $44(2)$ & $<0.001$ & $2184(100)$ \\
\hline$\beta$-blockers, $n(\%)$ & $153(60)$ & $380(20)$ & $<0.001$ & $2184(100)$ \\
\hline Ivabradine, $n(\%)$ & $1(0.4)$ & $2(0.1)$ & 0.788 & $2184(100)$ \\
\hline Digitalis glycoside, $n(\%)$ & $10(4)$ & $9(1)$ & $<0.001$ & $2184(100)$ \\
\hline Calcium blockers (nondihydropyridine), $n(\%)$ & $11(4)$ & $27(1)$ & 0.002 & $2184(100)$ \\
\hline Calcium blockers (dihydropyridine), $n(\%)$ & $65(26)$ & $196(10)$ & $<0.001$ & $2184(100)$ \\
\hline$\alpha$-adrenergic blockers, $n(\%)$ & $39(15)$ & $79(4)$ & $<0.001$ & $2184(100)$ \\
\hline Thiazide or thiazidelike diuretics, $n(\%)$ & $28(11)$ & $122(6)$ & 0.009 & $2184(100)$ \\
\hline Loop diuretics, $n(\%)$ & $100(39)$ & $85(4)$ & $<0.001$ & $2184(100)$ \\
\hline Statins, $n(\%)$ & $123(48)$ & $227(12)$ & $<0.001$ & $2184(100)$ \\
\hline Acetylsalicylic acid, $n(\%)$ & $76(30)$ & $182(9)$ & $<0.001$ & $2184(100)$ \\
\hline Second antiplatelet drug, $n(\%)$ & $20(8)$ & $19(1)$ & $<0.001$ & $2184(100)$ \\
\hline Low-molecular-weight heparin, $n(\%)$ & $28(11)$ & $113(6)$ & 0.003 & $2184(100)$ \\
\hline Vitamin $\mathrm{K}$ antagonists, $n(\%)$ & $22(9)$ & $25(1)$ & $<0.001$ & $2184(100)$ \\
\hline Direct oral anticoagulants, $n(\%)$ & $55(22)$ & $52(3)$ & $<0.001$ & $2184(100)$ \\
\hline Insulin, $n(\%)$ & $39(15)$ & $92(5)$ & $<0.001$ & $2184(100)$ \\
\hline Metformin, $n(\%)$ & $52(20)$ & $170(9)$ & $<0.001$ & $2184(100)$ \\
\hline Sodium-glucose cotransporter-2 inhibitors, $n(\%)$ & $11(4)$ & $16(1)$ & $<0.001$ & $2184(100)$ \\
\hline Oral antidiabetics other than mentioned above, $n(\%)$ & $30(12)$ & $59(3)$ & $<0.001$ & $2184(100)$ \\
\hline Oral corticosteroid, $n(\%)$ & $9(4)$ & $83(4)$ & 0.680 & $2184(100)$ \\
\hline Immunosuppression other than corticosteroid, $n(\%)$ & $8(3)$ & $65(3)$ & 0.993 & $2184(100)$ \\
\hline Home oxygen therapy or ventilation $n(\%)$ & $4(1.6)$ & $4(0.2)$ & 0.005 & $2184(100)$ \\
\hline Hemodialysis, $n(\%)$ & $21(8)$ & $37(2)$ & $<0.001$ & $2184(100)$ \\
\hline
\end{tabular}

Patients with a history of HF reported dyspnea and chest pain on admission more frequently and their physical examination revealed more abnormalities (lower oxygen saturation on room air, wheezing, pulmonary congestion, and peripheral edema than patients from the non-HF group (see Table 2). 
Table 2. Patient-reported symptoms, vital signs, and abnormalities measured during physical examination at hospital admission in the studied cohorts.

\begin{tabular}{|c|c|c|c|c|}
\hline Variables, Units & $\begin{array}{c}\text { HF Group } \\
n=255\end{array}$ & $\begin{array}{c}\text { Non-HF Group } \\
n=1929\end{array}$ & $p$ & $\begin{array}{c}\text { Available Data, } \\
n(\%)\end{array}$ \\
\hline \multicolumn{5}{|c|}{ Patient-Reported Symptoms } \\
\hline Cough, $n(\%)$ & $67(26)$ & $581(30)$ & 0.234 & $2184(100)$ \\
\hline Dyspnea, $n(\%)$ & $144(57)$ & $777(40)$ & $<0.001$ & $2184(100)$ \\
\hline Chest pain, $n(\%)$ & $29(11)$ & $134(7)$ & 0.016 & $2184(100)$ \\
\hline Hemoptysis, $n(\%)$ & $4(2)$ & $11(1)$ & 0.158 & $2184(100)$ \\
\hline Smell dysfunction, $n(\%)$ & $4(2)$ & $72(4)$ & 0.112 & $2184(100)$ \\
\hline Taste dysfunction, $n(\%)$ & $5(2)$ & $61(3)$ & 0.391 & $2184(100)$ \\
\hline Abdominal pain, $n(\%)$ & $16(6)$ & $131(7)$ & 0.860 & $2184(100)$ \\
\hline Diarrhea, $n(\%)$ & $16(6)$ & $111(6)$ & 0.848 & $2184(100)$ \\
\hline Vomiting, $n(\%)$ & $11(4)$ & $87(4)$ & 0.887 & $2184(100)$ \\
\hline \multicolumn{5}{|c|}{ Measured Vital Signs } \\
\hline Body temperature, ${ }^{\circ} \mathrm{C}$ & $36.9 \pm 0.9$ & $37.0 \pm 0.9$ & 0.182 & $1186(54)$ \\
\hline Heart rate, beats/minute & $85 \pm 19$ & $86 \pm 16$ & 0.364 & $1672(77)$ \\
\hline Systolic blood pressure, $\mathrm{mmHg}$ & $133 \pm 26$ & $132 \pm 22$ & 0.494 & $1169(76)$ \\
\hline $\mathrm{SpO} 2$ on room air, $\%$ & $90 \pm 10$ & $92 \pm 8$ & 0.001 & $1263(58)$ \\
\hline SpO2 on oxygen supplementation, $\%$ & $94 \pm 6$ & $95 \pm 6$ & 0.420 & $824(38)$ \\
\hline \multicolumn{5}{|c|}{ Abnormalities Detected during Physical Examination } \\
\hline Wheezing, $n(\%)$ & $59(23)$ & $160(8)$ & $<0.001$ & $2184(100)$ \\
\hline Pulmonary congestion, $n(\%)$ & $85(33)$ & $282(15)$ & $<0.001$ & $2184(100)$ \\
\hline Peripheral oedema, $n(\%)$ & $58(23)$ & $131(7)$ & $<0.001$ & $2184(100)$ \\
\hline
\end{tabular}

Patients with a history of HF were characterized by higher levels of cardiac (NTproBNP, troponin I) and inflammatory (leucocytes, procalcitonin, IL-6) biomarkers as well as higher potassium, urea, uric acid, bilirubin levels, and lower number of lymphocytes, hemoglobin concentration, and eGFR (see Table 3).

Table 3. Laboratory parameters measured on admission in the studied cohorts.

\begin{tabular}{lcccc}
\hline \multicolumn{1}{c}{ Variables, Units } & $\begin{array}{c}\text { HF Group } \\
\boldsymbol{n}=\mathbf{2 5 5}\end{array}$ & $\begin{array}{c}\text { Non-HF Group } \\
\boldsymbol{n}=\mathbf{1 9 2 9}\end{array}$ & $\boldsymbol{p}$ & Available Data, $\boldsymbol{n}$ (\%) \\
\hline Leukocytes, $10^{3} / \mu \mathrm{L}$ & $7.7(5.8-10.6)$ & $7.3(5.3-10.3)$ & 0.040 & $2050(94)$ \\
\hline Lymphocytes, $10^{3} / \mu \mathrm{L}$ & $0.9(0.6-1.4)$ & $1.0(0.7-1.4)$ & 0.007 & $1296(59)$ \\
\hline Neutrophils, $10^{3} / \mu \mathrm{L}$ & $5.7(3.8-9.1)$ & $5.5(3.4-8.2)$ & 0.058 & $1299(59)$ \\
\hline Hemoglobin, $\mathrm{g} / \mathrm{dL}$ & $12.0 \pm 2.4$ & $13.1 \pm 2.2$ & $<0.001$ & $2050(94)$ \\
\hline Sodium, $\mathrm{mmol} / \mathrm{L}$ & & Electrolytes, Inflammatory & & $2032(93)$ \\
\hline Potassium, $\mathrm{mmol} / \mathrm{L}$ & $4.3 \pm 0.8$ & $138 \pm 5$ & 0.958 & $<0.001$ \\
\hline CRP, $\mathrm{mg} / \mathrm{L}$ & $46(13-102)$ & $4.1 \pm 0.6$ & 0.686 & $2039(93)$ \\
\hline Procalcitonin, $\mathrm{ng} / \mathrm{mL}$ & $0.15(0.06-0.57)$ & $49(13-117)$ & $<0.001$ & $1475(68)$ \\
\hline
\end{tabular}


Table 3. Cont.

\begin{tabular}{lcccc}
\hline \multicolumn{1}{c}{ Variables, Units } & $\begin{array}{c}\text { HF Group } \\
\boldsymbol{n}=\mathbf{2 5 5}\end{array}$ & $\begin{array}{c}\text { Non-HF Group } \\
\boldsymbol{n}=\mathbf{1 9 2 9}\end{array}$ & $\boldsymbol{p}$ & Available Data, $\boldsymbol{n}$ (\%) \\
\hline IL-6, pg/mL & $28(11-59)$ & $16(6-44)$ & 0.002 & $702(32)$ \\
\hline Ferritin, $\mathrm{ng} / \mathrm{mL}$ & $557(197-1212)$ & $602(297-1150)$ & 0.309 & $969(44)$ \\
\hline Urea, $\mathrm{mg} / \mathrm{dL}$ & & Biochemistry & $1859(85)$ \\
\hline eGFR, $\mathrm{ml} / \mathrm{min} / 1.73 \mathrm{~m}^{2}$ & $64(44-101)$ & $36(25-57)$ & $<0.001$ & $1958(90)$ \\
\hline Albumin, g/L & $53 \pm 31$ & $78 \pm 34$ & $<0.001$ & $665(30)$ \\
\hline Uric acid, $\mathrm{mg} / \mathrm{dL}$ & $3.0 \pm 0.6$ & $3.1 \pm 0.6$ & 0.132 & $<23(29)$ \\
\hline Bilirubin, $\mathrm{mg} / \mathrm{dL}$ & $6.7(5.4-9.2)$ & $5.2(3.9-6.7)$ & $<0.001$ & $1408(64)$ \\
\hline GGTP, U/L & $0.7(0.5-1.1)$ & $0.6(0.5-0.8)$ & $<0.001$ & $1352(62)$ \\
\hline & $43(24-97)$ & $43(24-86)$ & 0.916 & $379(17)$ \\
\hline NT-proBNP, pg/mL & $6496(2255-15,881)$ & $551(160-2441)$ & & $1174(64)$ \\
\hline Troponin I, ng/L & $55(23-157)$ & $11(4-36)$ & $<0.001$ & $<0.001$ \\
\hline
\end{tabular}

3.2. The Association of HF History with In-Hospital Course and Applied Treatment and Procedures during Hospitalization

There were no statistically significant differences between groups in relation to the number of patients admitted to ICU and ventilated mechanically. However, HF patients were characterized by longer hospitalization and more often worsened during treatment (43\% vs. $24 \%, p<0.001$ ), including the development of shock, myocardial infarction (diagnosed according to Fourth Universal Definition of Myocardial Infarction [27]), and acute HF. These patients more often required passive oxygen therapy, noninvasive ventilation, use of catecholamines, loop diuretics, amiodarone, nitroglycerine, direct oral anticoagulants, acetylsalicylic acid, antibiotics, and interventions like coronary angiography, coronary revascularization, and hemodialysis (see Table 4).

Table 4. In-hospital course and therapies applied during the hospitalization in the studied cohorts.

\begin{tabular}{|c|c|c|c|c|}
\hline Variables, Units & $\begin{array}{l}\text { HF Group } \\
\quad n=255\end{array}$ & $\begin{array}{c}\text { Non-HF Group } \\
n=1929\end{array}$ & $p$ & $\begin{array}{c}\text { Available Data, } \\
n(\%)\end{array}$ \\
\hline \multicolumn{5}{|c|}{ In-hospital Course } \\
\hline Duration of hospitalization, days & $13(4-21)$ & $9(2-15)$ & $<0.001$ & $2184(100)$ \\
\hline Admission at intensive care unit, $n(\%)$ & $33(13)$ & $181(9)$ & 0.092 & $2184(100)$ \\
\hline Shock, $n(\%)$ & $38(15)$ & $150(8)$ & $<0.001$ & $2184(100)$ \\
\hline Myocardial infarction, $n(\%)$ & $10(4)$ & $16(1)$ & $<0.001$ & $2184(100)$ \\
\hline Thromboembolic disease, $n(\%)$ & $10(4)$ & $59(3)$ & 0.459 & $2184(100)$ \\
\hline Stroke, $n(\%)$ & $7(3)$ & $37(2)$ & 0.377 & $2184(100)$ \\
\hline Acute HF, $n(\%)$ & $53(21)$ & $23(1)$ & $<0.001$ & $2184(100)$ \\
\hline \multicolumn{5}{|c|}{ Applied Treatment and Procedures } \\
\hline Passive oxygen therapy, $n(\%)$ & $109(43)$ & $654(34)$ & 0.006 & $2181(100)$ \\
\hline Non-invasive ventilation, $n(\%)$ & $37(15)$ & $136(7)$ & $<0.001$ & $2181(100)$ \\
\hline
\end{tabular}


Table 4. Cont.

\begin{tabular}{|c|c|c|c|c|}
\hline Variables, Units & $\begin{array}{l}\text { HF Group } \\
\quad n=255\end{array}$ & $\begin{array}{c}\text { Non-HF Group } \\
n=1929\end{array}$ & $p$ & $\begin{array}{c}\text { Available Data, } \\
n(\%)\end{array}$ \\
\hline Mechanical ventilation, $n(\%)$ & $33(13)$ & $182(9)$ & 0.098 & $2184(100)$ \\
\hline Duration of mechanical ventilation, days & $8(2-15)$ & $9(4-17)$ & 0.176 & $197(9)$ \\
\hline Therapy with catecholamines, $n(\%)$ & $45(18)$ & $173(9)$ & $<0.001$ & $2184(100)$ \\
\hline Therapy with loop diuretics, $n(\%)$ & $104(41)$ & $229(12)$ & $<0.001$ & $2184(100)$ \\
\hline Extracorporeal membrane oxygenation, $n(\%)$ & $1(0.4)$ & $22(1.1)$ & 0.439 & $2184(100)$ \\
\hline Coronary angiography, $n(\%)$ & $12(5)$ & $18(1)$ & $<0.001$ & $2184(100)$ \\
\hline Coronary revascularization, $n(\%)$ & $11(4)$ & $20(1)$ & 0.001 & $2184(100)$ \\
\hline Hemodialysis, $n(\%)$ & $17(7)$ & $55(3)$ & 0.003 & $2184(100)$ \\
\hline Amiodarone, $n(\%)$ & $17(7)$ & $39(2)$ & $<0.001$ & $2184(100)$ \\
\hline Nitroglycerine (i.v.), $n(\%)$ & $7(3)$ & $12(1)$ & 0.002 & $2184(100)$ \\
\hline Low-molecular-weight heparin, $n(\%)$ & $160(63)$ & $1105(57)$ & 0.111 & $2184(100)$ \\
\hline Unfractionated heparin, $n(\%)$ & $12(5)$ & $110(6)$ & 0.613 & $2184(100)$ \\
\hline Direct oral anticoagulants, $n(\%)$ & $29(11)$ & $6(3)$ & $<0.001$ & $2184(100)$ \\
\hline Vitamin $\mathrm{K}$ antagonists, $n(\%), n(\%)$ & $5(2)$ & $15(1)$ & 0.130 & $2184(100)$ \\
\hline Acetylsalicylic acid, $n(\%)$ & $81(32)$ & $285(15)$ & $<0.001$ & $2184(100)$ \\
\hline Thrombolytic therapy, $n(\%)$ & $1(0.4)$ & $13(0.7)$ & 0.911 & $2184(100)$ \\
\hline Systemic corticosteroid, $n(\%)$ & $129(51)$ & $967(50)$ & 0.943 & $2184(100)$ \\
\hline Convalescent plasma, $n(\%)$ & $33(13)$ & $206(11)$ & 0.327 & $2184(100)$ \\
\hline Hydroxychloroquine/chloroquine, $n(\%)$ & $0(0)$ & $9(1)$ & 0.567 & $2184(100)$ \\
\hline Tocilizumab, $n(\%)$ & $1(0.4)$ & $24(1.2)$ & 0.374 & $2184(100)$ \\
\hline Remdesivir, $n(\%)$ & $38(15)$ & $305(16)$ & 0.777 & $2184(100)$ \\
\hline Antibiotic, $n(\%)$ & $168(66)$ & $1073(56)$ & 0.002 & $2184(100)$ \\
\hline Prone positioning, $n(\%)$ & $22(9)$ & $182(9)$ & 0.761 & $2184(100)$ \\
\hline
\end{tabular}

The predictors of acute HF were higher age and history of HF (see Table 5). The multivariable model included variables with a $p$-value $<0.001$ in the univariable model.

Table 5. Predictors of acute HF during hospitalization in patients with COVID-19.

\begin{tabular}{lccc}
\hline Variables, Units & Univariable Models & Multivariable Model & \multicolumn{1}{c}{ OR (95\% CI) } \\
\hline Age, per 5 years & OR (95\% CI) & $1.23(1.11-1.36)$ & - \\
\hline Arterial hypertension, yes/no & $1.39(1.27-1.51)$ & - & - \\
\hline Diabetes mellitus, yes/no & $3.82(2.24-6.54)$ & - & - \\
\hline History of heart failure, yes/no & $2.73(1.72-4.34)$ & $11.42(6.49-20.10)$ & $<0.001$ \\
\hline History of myocardial infarction, yes/no & $21.74(13.05-36.23)$ & $1.67(0.95-2.94)$ & 0.074 \\
\hline Atrial fibrillation/flutter, yes/no & $6.12(3.71-10.10)$ & - & - \\
\hline
\end{tabular}


Table 5. Cont.

\begin{tabular}{lccc}
\hline Variables, Units & Univariable Models & Multivariable Model \\
\hline Valvular heart disease, yes/no & $5.58(3.00-10.38)$ & - & - \\
\hline Peripheral artery disease, yes/no & $3.84(1.96-7.53)$ & - & - \\
\hline Chronic kidney disease, yes/no & $4.53(2.75-7.46)$ & - & - \\
\hline
\end{tabular}

Chi2 $=171, p<0.001$. OR: odds ratio, CI: confidence interval.

\subsection{Outcome}

There was high mortality in the studied population with $326 / 2184(15 \%)$ deaths during hospitalization, 546/2184 (25\%) after three months, and 578/1491 (26\%) after six months from admission. We found a relationship between history of HF before hospitalization and mortality in COVID-19 patients in relation to all endpoints (see Figures 1 and 2). The HF group had significantly higher mortality assessed in hospital: 89 (35\%) vs. $237(12 \%)$, 3-month mortality: 132 (53\%) vs. 413 (22\%) and 6-month mortality: 141 (72\%) vs. $436(47 \%)$, all $p<0.001$.
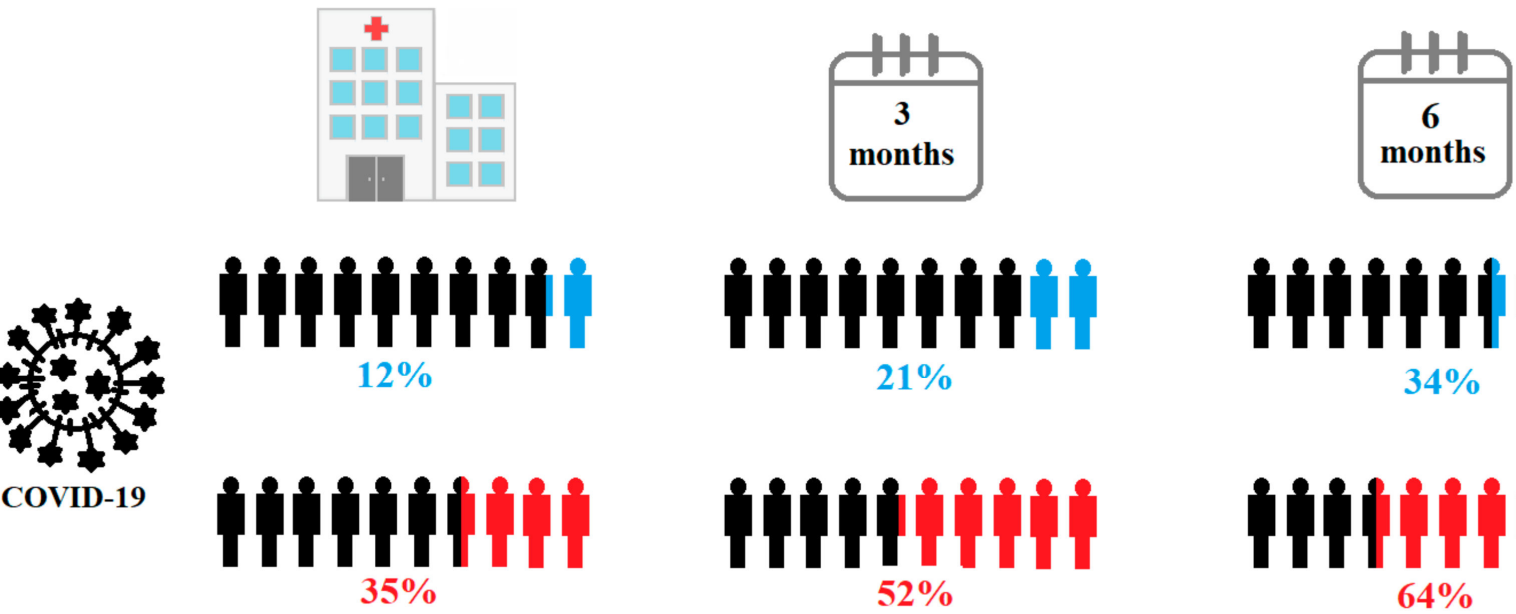

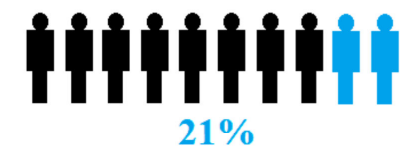

Milihilit

$52 \%$

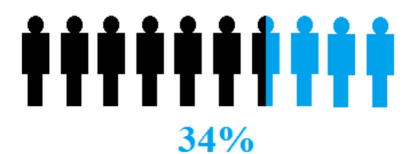

minimin

$64 \%$

$$
\text { Legend: } \begin{aligned}
& \text { mortality } \\
& \text { in no-HF group }
\end{aligned}
$$

Figure 1. The all-cause mortality in the studied groups.

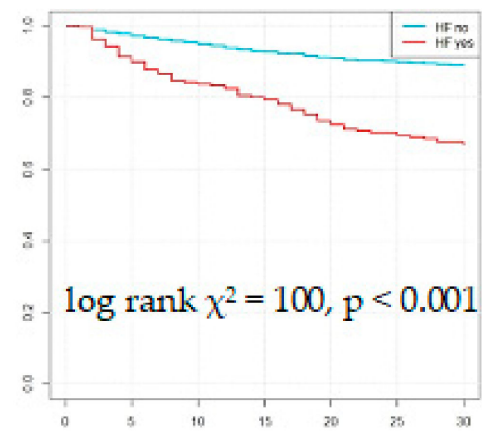

A. Overall in-hospital mortality.

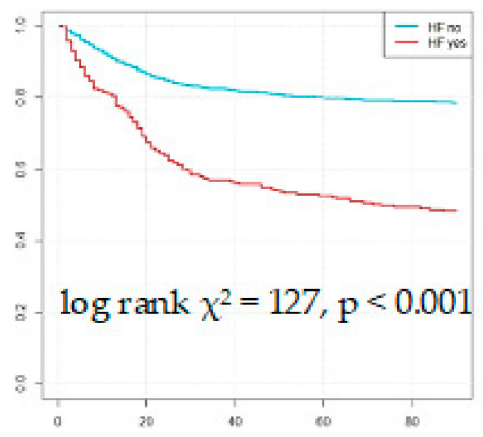

B. All-cause 3-months mortality.

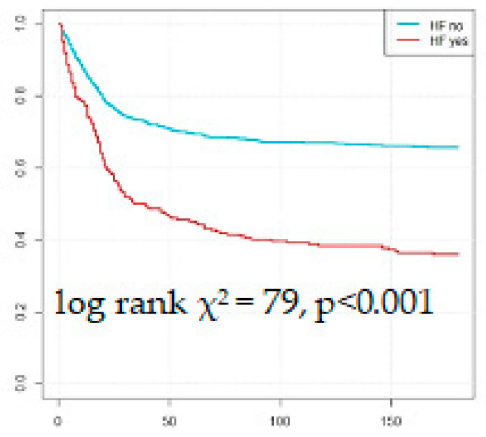

C. All-cause 6-months mortality.

Figure 2. Kaplan-Meier curves for all-cause mortality in studied groups. 
After the adjustment of variables that appeared to be significant predictors in univariate Cox analysis, history of HF was revealed to be a predictor of higher risk for three months mortality (see Tables 6 and 7).

Table 6. Predictors of 3-month mortality-univariable model.

\begin{tabular}{lccc}
\hline \multicolumn{1}{c}{ Variables } & Units & Univariable Model \\
\cline { 3 - 4 } & & HR (95\% CI) & $p$ \\
\hline Age & 5 years & $1.31(1.27-1.35)$ & $<0.001$ \\
History of heart failure & yes/no & $2.95(2.42-3.59)$ & $<0.001$ \\
Gender & Male & $1.53(1.29-1.82)$ & $<0.001$ \\
Diabetes mellitus & yes/no & $1.89(1.57-2.26)$ & $<0.001$ \\
Arterial hypertension & yes/no & $2.16(1.82-2.58)$ & $<0.001$ \\
Chronic obstructive pulmonary disease & yes/no & $2.59(1.87-3.59)$ & $<0.001$ \\
Previous stroke/transient ischemic attack & yes/no & $1.80(1.39-2.33)$ & $<0.001$ \\
Chronic kidney disease & yes/no & $2.43(1.97-3.00)$ & $<0.001$ \\
Malignancy & yes/no & $2.42(1.93-3.03)$ & $<0.001$ \\
C-reactive protein & Ln 1 mg/L & $1.49(1.39-1.60)$ & $<0.001$ \\
Hemoglobin & $1 \mathrm{~g} / \mathrm{dL}$ & $0.87(0.84-0.90)$ & $<0.001$ \\
\hline
\end{tabular}

Table 7. Predictors of 3-month mortality-multivariable model.

\begin{tabular}{|c|c|c|c|c|c|}
\hline \multirow{2}{*}{ Variables } & \multirow{2}{*}{ Units } & \multicolumn{4}{|c|}{ Multivariable Models } \\
\hline & & HR (95\% CI) & Wald's Statistics & $p$ & Chi2 $(p)$ \\
\hline Age & 5 years & $1.29(1.25-1.33)$ & 269 & $<0.001$ & \\
\hline History of heart failure & yes/no & $1.54(1.25-1.88)$ & 17 & $<0.001$ & $416(<0.001)$ \\
\hline Gender & Male & $1.53(1.29-1.82)$ & 20 & $<0.001$ & \\
\hline History of heart failure & yes/no & $2.86(2.35-3.48)$ & 109 & $<0.001$ & $114(<0.001)$ \\
\hline Age & 5 years & $1.32(1.28-1.37)$ & 283 & $<0.001$ & \\
\hline Gender & Male & $1.82(1.53-2.17)$ & 45 & $<0.001$ & $462(<0.001)$ \\
\hline History of heart failure & yes/no & $1.48(1.21-1.81)$ & 14 & $<0.001$ & \\
\hline Age & 5 years & $1.32(1.27-1.36)$ & 250 & $<0.001$ & \\
\hline Gender & Male & $1.71(1.43-2.04)$ & 36 & $<0.001$ & \\
\hline Diabetes mellitus & yes/no & $1.20(0.99-1.44)$ & 3.6 & 0.059 & \\
\hline Arterial hypertension & yes/no & $0.91(0.75-1.11)$ & 0.9 & 0.346 & \\
\hline COPD & yes/no & $1.40(1.00-1.96)$ & 3.9 & 0.049 & $504(<0.001)$ \\
\hline Previous stroke/TIA & yes/no & $0.93(0.71-1.21)$ & 0.3 & 0.579 & \\
\hline Chronic kidney disease & yes/no & $1.40(1.11-1.75)$ & 8.1 & 0.004 & \\
\hline Malignancy & yes/no & $1.87(1.49-2.35)$ & 29 & $<0.001$ & \\
\hline History of heart failure & yes/no & $1.31(1.05-1.63)$ & 5.78 & 0.016 & \\
\hline Age & 5 years & $1.30(1.26-1.35)$ & 204 & $<0.001$ & \\
\hline Gender & Male & $1.59(1.33-1.91)$ & 26 & $<0.001$ & \\
\hline Diabetes mellitus & yes/no & $1.12(0.92-1.35)$ & 1.3 & 0.252 & \\
\hline Arterial hypertension & yes/no & $0.93(0.76-1.12)$ & 0.6 & 0.433 & \\
\hline COPD & yes/no & $1.38(0.98-1.93)$ & 3.4 & 0.065 & \\
\hline Previous stroke/TIA & yes/no & $0.98(0.74-1.29)$ & 0.03 & 0.87 & $571(<0.001)$ \\
\hline Chronic kidney disease & yes/no & $1.30(1.03-1.65)$ & 4.7 & 0.03 & \\
\hline Malignancy & yes/no & $1.86(1.47-2.35)$ & 27 & $<0.001$ & \\
\hline CRP & $\mathrm{Ln} 1 \mathrm{mg} / \mathrm{L}$ & $1.42(1.32-1.52)$ & 93 & $<0.001$ & \\
\hline Hemoglobin & $1 \mathrm{~g} / \mathrm{dL}$ & $0.94(0.91-0.98)$ & 9.2 & 0.002 & \\
\hline History of heart failure & yes/no & $1.35(1.08-1.69)$ & 7 & 0.008 & \\
\hline
\end{tabular}

HR: hazard ratio; CI: confidence interval; COPD: chronic obstructive pulmonary disease; TIA: transient ischemic attack; CKD: chronic kidney disease; CRP: C-reactive protein; Ln: natural logarithm.

Among patients with a history of HF, significantly higher risk of three-month death was noted in patients $\leq 70$ years old and in women $\leq 70$ years old when compared with 
non-HF group. There were no differences in mortality risk in patients with a history of $\mathrm{HF}$ divided according to gender (see Figure 3).

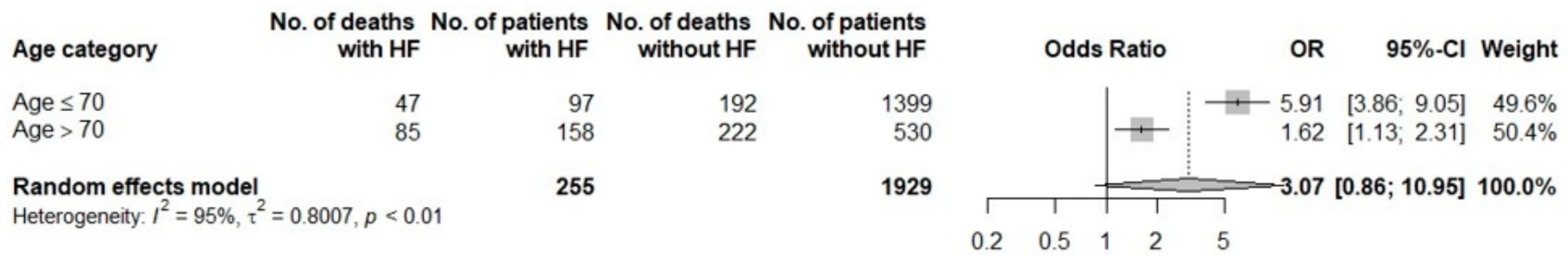

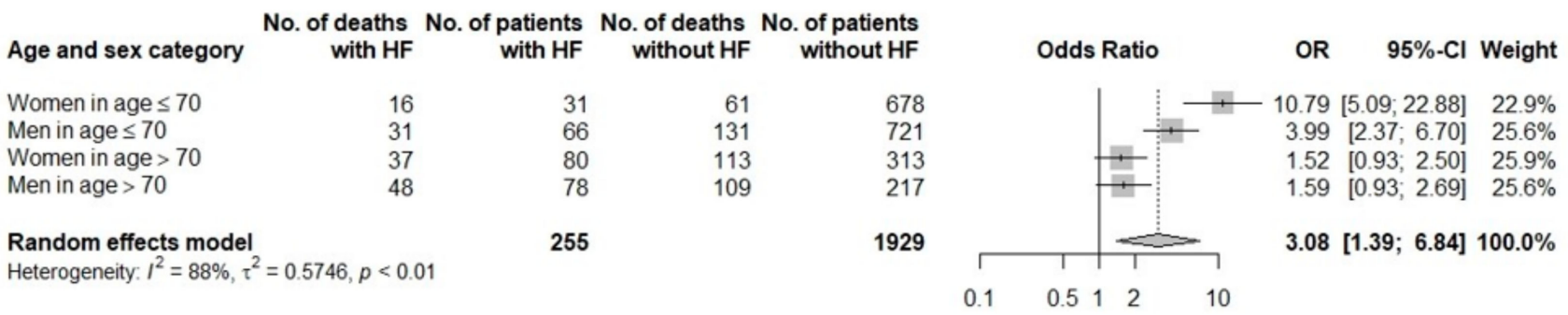

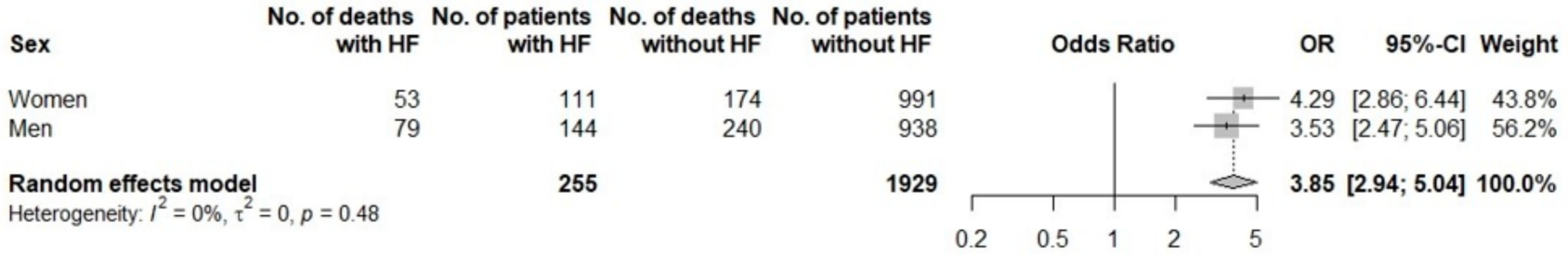

Figure 3. Forest plots for age, sex, and age/sex categories in patients with a history of heart failure vs. without history of heart failure.

Patients who developed acute HF during hospitalization had extremely high mortality: 54/76 (71\%) died during hospitalization, 60/76 (79\%) at three, and 61/70 (87\%) at six months. Analyzing HF-group with available left ventricular ejection fraction (LVEF) measurement (130/255 patients, 51\%), the mean LVEF was $46 \% \pm 16 \%$. There were 70 (54\%) patients with HF with preserved ejection fraction (HFpEF), 19 (15\%) with HF with mildly reduced ejection fraction (HFmrEF) and $41(32 \%)$ with HF with reduced ejection fraction (HFrEF). In a subgroup analysis, patients with HFrEF and HFmrEF had higher in-hospital, 3-, and 6-month mortality, compared to patients with HFpEF (see Table 8).

Table 8. Outcomes investigated in patients according to left ventricular ejection fraction.

\begin{tabular}{ccccc}
\hline Variables, Units & HFrEF & HFmrEF & HFpEF & $p$ \\
\hline In-hospital mortality, $n(\%)$ & $18(44)$ & $8(42)$ & $14(20)$ & 0.016 \\
\hline 3-month mortality, $n(\%)$ & $26(63)$ & $13(68)$ & $19(27)$ & $<0.001$ \\
\hline 6-month mortality, $n(\%)$ & $26(74)$ & $13(76)$ & $25(42)$ & 0.002 \\
\hline
\end{tabular}

HFrEF: heart failure with reduced ejection fraction; HFmrEF heart failure with mildly reduced ejection fraction; HFpEF: heart failure with preserved ejection fraction.

Similar findings are related to those patients that had to be taken to ICU as compared to those without ICU stay (see Tables 9 and 10). 
Table 9. Outcomes investigated in patients admitted to intensive care unit vs. those without intensive care unit stay.

\begin{tabular}{cccc}
\hline Variables, Units & $\begin{array}{c}\text { Intensive Care } \\
\text { Unit Stay }\end{array}$ & $\begin{array}{c}\text { Without Intensive } \\
\text { Care Unit Stay }\end{array}$ & $p$ \\
\hline In-hospital mortality, $n(\%)$ & $118(55)$ & $208(11)$ & $<0.001$ \\
3-month mortality, $n(\%)$ & $132(62)$ & $414(21)$ & $<0.001$ \\
6-month mortality, $n(\%)$ & $134(71)$ & $444(34)$ & $<0.001$ \\
\hline
\end{tabular}

Table 10. Outcomes investigated in patients admitted to intensive care unit according to history of heart failure.

\begin{tabular}{cccc}
\hline Variables, Units & HF Group & Non-HF Group & $p$ \\
\hline In-hospital mortality, $n(\%)$ & $25(76)$ & $93(51)$ & 0.009 \\
3-month mortality, $n(\%)$ & $27(82)$ & $105(58)$ & 0.010 \\
6-month mortality, $n(\%)$ & $27(87)$ & $17(68)$ & 0.033 \\
\hline
\end{tabular}

\section{Discussion}

Our study for the first time analyzed not only the in-hospital course but also longterm mortality in this group of patients. The presented study, performed among a large cohort of hospitalized patients with COVID-19, showed how history of HF affects the in-hospital course and especially long-term follow-up. One-third of HF patients died during hospitalization and the next one-third died after hospital discharge in the next three months.

Older age, gender, and comorbidities are well-known risk factors of severe course of COVID-19. Patients with HF are usually elderly, with multiple underlying medical conditions increasing their risk of complications and mortality after SARS-CoV-2 infection [28-30]. Especially cardiovascular diseases and their risk factors (e.g., hypertension, diabetes mellitus, atrial fibrillation, ischemic heart disease) are common among HF patients [31]. However, HF remains independently associated with in-hospital mortality [24]. In our multivariable analyses, history of $\mathrm{HF}$ increased the risk of primary endpoint incidence from $30 \%$ to $83 \%$.

The human angiotensin converting enzyme 2 (ACE2) receptor is a gateway for SARSCoV-2 to enter cells. In the heart, the ACE2 receptors can be found on the surface of cardiomyocytes, cardiac fibroblasts, and endothelia of coronary arteries [32]. Recent studies showed increased levels of ACE2 in patients with HF, especially males consistently with the increased severity of COVID-19 among men [32-34].

Myocardial damage in COVID-19 can be caused by endothelial dysfunction and systemic inflammatory response, leading to plaque instability and prothrombotic state (type $1 \mathrm{MI}$ ), as well as by mismatch between oxygen supply and/or demand related with respiratory failure (type 2 MI) [35-38]. However, the role of SARS-CoV-2 in the direct myocardial injury ("myocarditis-like" effect) is still to be determined [36].

As reported in our manuscript, patients with a history of HF were characterized by higher levels of cardiac (NT-proBNP, troponin I) biomarkers and more often developed myocardial infarction. MI was diagnosed according to the Fourth Universal Definition of Myocardial Infarction [27]. However, troponin elevation can also be observed in a high number of clinical conditions, e.g., HF and a septic state [36,39]. It should be emphasized that troponin levels are mostly influenced by baseline characteristics, such as pre-existing HF or coronary artery disease [36,37]. In the study of 355 patients hospitalized with COVID19 , the levels of troponin were significantly higher in the group of patients with chronic coronary syndromes (CCS). Nevertheless, over $89 \%$ of significant troponin elevations in our sample population were nonspecific troponin leaks while only $11 \%$ had actual non-ST elevation [37].

Therefore, cardiac biomarkers elevation in COVID-19 may be sometimes misleading. Although myocardial injury has been shown to be associated with worse outcomes 
in COVID-19, a recent study revealed that troponin elevation had a prognostic value in non-CCS patients, while it was not predictive of poor outcomes in patients with CCS $[36,39]$ Further studies are necessary to determine the role of troponin elevation as a prognostic marker.

In our study, significantly more women and men died in the HF group, compared to those in the non-HF group, but it also showed that the ratio of deaths between women and men did not differ in both groups. It should be emphasized that a significantly higher proportion of deaths was in younger people and younger women in the HF group than in those without HF.

The renal impairment secondary to acute kidney injury is observed in up to $30 \%$ of patients with COVID-19 $[13,20]$. While cardiorenal syndrome is a spectrum of well-known disorders in HF, in our study, patients with HF had worse kidney function, but the presence of chronic kidney disease was not associated with the higher risk of acute HF [40]. In the multivariable model, the only risk factors for developing acute HF were more advanced age and a history of previous HF.

In-hospital mortality rate of HF patients hospitalized with COVID-19 presented in our study remains consistent with prior literature. A study based on the multicenter, multinational PCHF-COVICAV registry analyzed 1974 patients hospitalized with COVID19, HF was present in 256 patients. 1282 (65\%) of patients had cardiovascular disease and/or risk factors. In this study, in-hospital mortality in the HF group reached 36\% [24].

Furthermore, in the retrospective analysis of 6439 patients hospitalized in New York with COVID-19, the group of patients with HF was smaller ( $n=422 ; 7 \%)$. The most frequent comorbidities in the whole study group were hypertension (35\%), obesity (28\%), and diabetes mellitus (23\%). Likewise, compared with patients without HF, those with a history of HF were older, had a higher prevalence of comorbidities, and were receiving a greater number of medications. While total mortality was $26 \%$, the risk of mortality among patients with HF was significantly higher than in the group of patients without HF (40 vs. $25 \%$ ) [41].

In the study of 3080 patients with confirmed COVID-19, a group of $152(5 \%)$ had a previous history of HF. Similar to our study, patients with HF had more cardiovascular risk factors and comorbidities than patients without a history of HF, and the most common were hypertension (86\%), dyslipidemia (75\%), atrial fibrillation/flutter (52\%), and diabetes $(40 \%)$. The overall mortality rate was $21 \%$ and was significantly higher in patients with previous HF (49 vs. 19\%) [42].

However, the aforementioned studies analyzed HF patients already hospitalized with COVID-19; therefore, a certain selection bias cannot be excluded. In the study of 31,051 ambulatory patients with COVID-19, 20\% of them had pre-existing HF and those had a 30-day mortality and 30-day admission rate of 5\% and $19 \%$, respectively. The overall 30 -day mortality and 30-day admission rates were $2 \%$ and $10 \%$, respectively. Patients with HF had higher comorbidity burden than the group without HF-they were more likely to be smokers (29\% vs. $13 \%$ ), have hypertension ( $84 \%$ vs. $50 \%$ ), diabetes (54\% vs. $28 \%$ ), myocardial infarction ( $44 \%$ vs. $120 \%)$, and atrial fibrillation ( $29 \%$ vs. $4 \%$ ) [43]. Thus, the profile of comorbidities seems similar in the groups of hospitalized and ambulatory HF patients.

In our study group, the frequency of comorbidities was similar to that reported in the prior literature-the most common concomitant disorders were hypertension (84\%), atrial fibrillation (53\%), diabetes mellitus (48\%), and chronic kidney disease (36\%) [41,42]. However, the incidence of atrial fibrillation among hospitalized HF patients is much higher in our study than in ambulatory patients [43]. Contrarily, the prevalence of cardiovascular disease and/or risk factors as well as HF were higher in the PCHF-COVICAV registry [24].

Before the COVID-19 outbreak, the prognosis in HF was unsatisfactory with the one year-mortality of patients after HF diagnosis estimated at $24 \%$ [5]. In-hospital mortality varied from $12 \%$ to $19 \%$, depending on the study [6]. As our study revealed, SARS-CoV-2 infection significantly worsens in-hospital course and long-term prognosis of HF patients 
with three-month mortality two times higher than in HF patients without COVID-19. In the study assessing long-term outcomes after HF hospitalization during the COVID-19 pandemic in the group without SARS-CoV-2 infection, the median follow-up was 622 days. The number of patients that died post-discharge for 2020 and 2019 cohorts was 172 (44\%) and $321(34 \%)$, respectively [44]. Interestingly, a recent study showed that during the COVID-19 outbreak, there was a significant decrease in acute admissions, including HF, to the cardiology and emergency departments. While the in-hospital mortality in 2020 and 2019 was similar (3.6\% vs. 3.9\%, respectively), the death rates of patients admitted to emergency departments were four times higher [23].

\section{Limitations}

Our study is limited by its retrospective, single-center character, which may limit its generalizability. Moreover, the study protocol did not include assessment of LVEF, and only small group of patients were evaluated with transthoracic echocardiography (TTE) due to safety reasons and from certain fraction of patients previous LVEF measurement was not available. Therefore, we are not able to stratify all HF population in relation to LVEF. Furthermore, the protocol of our study did not distinguish types of MI.

Thus, further prospective studies are required, not only to confirm the impact of SARSCoV-2 infections on long-term outcomes of patients with HF but also to assess quality of medical care during COVID-19 pandemic. While the pathophysiology of COVID-19 and the risk of HF are still insufficiently understood, the impact of comorbidities on survival of patients with HF and COVID-19 should be also examined. Further analyses are needed in order to explain higher mortality among patients with $\mathrm{HF}$, regardless of the presence of SARS-CoV-2 infection.

\section{Conclusions}

This retrospective study demonstrates high in-hospital mortality and poor long-term outcomes in patients with a history of HF hospitalized with COVID-19. The development of acute HF during hospitalization is associated with extremely high in-hospital and longterm mortality. Therefore, HF patients should be well and closely controlled; they should be treated both during hospitalization and afterward.

Author Contributions: Conceptualization, M.S., K.R., M.P., K.M. and E.A.J.; formal analysis, M.S. and T.S.; investigation, M.S., B.A., A.D., J.D., J.G.-D., M.J., K.K., K.K.-P., B.K., A.K., A.L., W.L., A.M.-W., L.P.-S., M.R.-B., J.M.S., J.S., A.Z.-T. and M.P.; methodology, M.S., T.S. and E.A.J.; project administration, M.S., M.P., K.M. and E.A.J.; supervision, M.P. and K.M.; visualization, M.S., T.S. and J.M.S.; writingoriginal draft, M.S., K.R., T.S., J.M.S. and E.A.J.; writing-review and editing, M.S., K.R., T.S., B.A., A.D., J.D., J.G.-D., M.J., K.K., K.K.-P., B.K., A.K., A.L., W.L., A.M.-W., L.P.-S., M.R.-B., J.M.S., J.S., A.Z.-T., M.P., K.M. and E.A.J. All authors have contributed substantially to this work. All authors have read and agreed to the published version of the manuscript.

Funding: The fundings from reserve of Rector for Scientific Affairs.

Institutional Review Board Statement: The study was conducted according to the guidelines of the Declaration of Helsinki and approved by the Bioethics Committee of Wroclaw Medical University, Wroclaw, Poland (Signature number: KB-444/2021).

Informed Consent Statement: The routine data were collected retrospectively; therefore, written informed consent to participate in the study was not required. The Bioethics Committee approved the publication of anonymized data.

Data Availability Statement: The data sets used and analyzed during the current study are available from the corresponding author on reasonable request.

Conflicts of Interest: The authors declare no conflict of interest. 


\section{References}

1. GBD 2016 Disease and Injury Incidence and Prevalence Collaborators. Global, regional, and national incidence, prevalence, and years lived with disability for 328 diseases and injuries for 195 countries, 1990-2016: A systematic analysis for the Global Burden of Disease Study 2016. Lancet 2017, 390, 1211-1259. [CrossRef]

2. Groenewegen, A.; Rutten, F.H.; Mosterd, A.; Hoes, A.W. Epidemiology of heart failure. Eur. J. Heart Fail. 2020, 22, 1342-1356. [CrossRef]

3. Benjamin, E.J.; Virani, S.S.; Callaway, C.W.; Chamberlain, A.M.; Chang, A.R.; Cheng, S.; Chiuve, S.E.; Cushman, M.; Delling, F.N.; Deo, R.; et al. Heart Disease and Stroke Statistics-2018 Update: A Report from the American Heart Association. Circulation 2018, 137, e67-e492. [CrossRef]

4. Nessler, J.; Siniarski, A.; Leszek, P.; Gumprecht, J.; Drożdż, J.; Kaźmierczak, J.; Witkowski, A.; Gackowski, A.; Jankowska, E.A.; Kukulski, T.; et al. Expert opinion of the Heart Failure Working Group of the Polish Cardiac Society on the use of dapagliflozin in the treatment of heart failure with reduced ejection fraction. Kardiol. Pol. 2021, 79, 363-370. [CrossRef]

5. Taylor, C.J.; Ordóñez-Mena, J.M.; Roalfe, A.K.; Lay-Flurrie, S.; Jones, N.; Marshall, T.; Hobbs, F.D.R. Trends in survival after a diagnosis of heart failure in the United Kingdom 2000-2017: Population based cohort study. BMJ 2019, 364, 1223. [CrossRef]

6. Sierpiński, R.; Sokolska, J.M.; Suchocki, T.; Koń, B.; Urbański, F.; Kruk, M.; Sokolski, M.; Ponikowski, P.; Jankowska, E.A. 10 year trends in hospitalization rates due to heart failure and related in-hospital mortality in Poland (2010-2019). ESC Heart Fail. 2020, 7, 3365-3373. [CrossRef]

7. World Health Organization. COVID-19 Explorer. Available online: https:/ / worldhealthorg.shinyapps.io/covid/ (accessed on 21 October 2021).

8. Jin, Y.; Yang, H.; Ji, W.; Wu, W.; Chen, S.; Zhang, W.; Duan, G. Virology, Epidemiology, Pathogenesis, and Control of COVID-19. Viruses 2020, 12, 372. [CrossRef]

9. $\quad$ Roth, G.A.; Emmons-Bell, S.; Alger, H.M.; Bradley, S.M.; Das, S.R.; de Lemos, J.A.; Gakidou, E.; Elkind, M.S.V.; Hay, S.; Hall, J.L.; et al. Trends in Patient Characteristics and COVID-19 In-Hospital Mortality in the United States during the COVID-19 Pandemic. JAMA Netw. Open 2021, 4, e218828. [CrossRef]

10. Gray, W.K.; Navaratnam, A.V.; Day, J.; Babu, P.; Mackinnon, S.; Adelaja, I.; Bartlett-Pestell, S.; Moulton, C.; Mann, C.; Batchelor, A.; et al. Variability in COVID-19 in-hospital mortality rates between national health service trusts and regions in England: A national observational study for the Getting It Right First Time Programme. EClinicalMedicine 2021, 35, 100859. [CrossRef]

11. Günster, C.; Busse, R.; Spoden, M.; Rombey, T.; Schillinger, G.; Hoffmann, W.; Weber-Carstens, S.; Schuppert, A.; Karagiannidis, C. 6-month mortality and readmissions of hospitalized COVID-19 patients: A nationwide cohort study of 8679 patients in Germany. PLOS ONE 2021, 16, e0255427. [CrossRef]

12. Streeck, H.; Schulte, B.; Kümmerer, B.M.; Richter, E.; Höller, T.; Fuhrmann, C.; Bartok, E.; Dolscheid-Pommerich, R.; Berger, M.; Wessendorf, L.; et al. Infection fatality rate of SARS-CoV2 in a super-spreading event in Germany. Nat. Commun. 2020, 11, 5829. [CrossRef]

13. Yang, X.; Yu, Y.; Xu, J.; Shu, H.; Xia, J.; Liu, H.; Wu, Y.; Zhang, L.; Yu, Z.; Fang, M.; et al. Clinical course and outcomes of critically ill patients with SARS-CoV-2 pneumonia in Wuhan, China: A single-centered, retrospective, observational study. Lancet Respir. Med. 2020, 8, 475-481. [CrossRef]

14. Gao, Y.-D.; Ding, M.; Dong, X.; Zhang, J.-J.; Azkur, A.K.; Azkur, D.; Gan, H.; Sun, Y.-L.; Fu, W.; Li, W.; et al. Risk factors for severe and critically ill COVID-19 patients: A review. Allergy 2021, 76, 428-455. [CrossRef]

15. Centers for Disease Control and Prevention. Provisional COVID-19 Death Counts by Sex, Age, and State. Available online: https: / / data.cdc.gov/NCHS/Provisional-COVID-19-Death-Counts-by-Sex-Age-and-S/9bhg-hcku (accessed on 21 October 2021).

16. Pollard, C.A.; Morran, M.P.; Nestor-Kalinoski, A.L. The COVID-19 pandemic: A global health crisis. Physiol. Genom. 2020, 52, 549-557. [CrossRef]

17. Jin, J.M.; Bai, P.; He, W.; Wu, F.; Liu, X.F.; Han, D.M.; Liu, S.; Yang, J.K. Gender Differences in Patients with COVID-19: Focus on Severity and Mortality. Front. Public Health 2020, 8, 152. [CrossRef]

18. Bader, F.; Manla, Y.; Atallah, B.; Starling, R.C. Heart failure and COVID-19. Heart Fail. Rev. 2020, 26, 1-10. [CrossRef]

19. Sokolski, M.; Sokolska, J.M.; Zymliński, R.; Biegus, J.; Banasiak, W.; Reczuch, K.; Ponikowski, P. Cardiac emergencies during the coronavirus disease 2019 pandemic in the light of the current evidence. Kardiol. Pol. 2020, 78, 818-824. [CrossRef]

20. Zhou, F.; Yu, T.; Du, R.; Fan, G.; Liu, Y.; Liu, Z.; Xiang, J.; Wang, Y.; Song, B.; Gu, X.; et al. Clinical course and risk factors for mortality of adult inpatients with COVID-19 in Wuhan, China: A retrospective cohort study. Lancet 2020, 395, 1054-1062. [CrossRef]

21. Kubica, J.; Ostrowska, M.; Stolarek, W.; Kasprzak, M.; Grzelakowska, K.; Kryś, J.; Kubica, A.; Adamski, P.; Podhajski, P.; Navarese, E.P.; et al. Impact of COVID-19 pandemic on acute heart failure admissions and mortality: A multicentre study (COV-HF-SIRIO 6 study). ESC Heart Fail 2021. advance online publication. [CrossRef]

22. Severino, P.; D'Amato, A.; Saglietto, A.; D'Ascenzo, F.; Marini, C.; Schiavone, M.; Ghionzoli, N.; Pirrotta, F.; Troiano, F.; Cannillo, M.; et al. Reduction in heart failure hospitalization rate during coronavirus disease 19 pandemic outbreak. ESC Heart Fail. 2020, 7, 4182-4188. [CrossRef]

23. Sokolski, M.; Gajewski, P.; Zymliński, R.; Biegus, J.; Berg, J.M.T.; Bor, W.; Braunschweig, F.; Caldeira, D.; Cuculi, F.; D’Elia, E.; et al. Impact of Coronavirus Disease 2019 (COVID-19) Outbreak on Acute Admissions at the Emergency and Cardiology Departments Across Europe. Am. J. Med. 2020, 134, 482-489. [CrossRef] 
24. Sokolski, M.; Trenson, S.; Sokolska, J.M.; D’Amario, D.; Meyer, P.; Poku, N.K.; Biering-Sørensen, T.; Lassen, M.C.H.; Skaarup, K.G.; Barge-Caballero, E.; et al. Heart failure in COVID-19: The multicentre, multinational PCHF-COVICAV registry. ESC Heart Fail. 2021. [CrossRef]

25. Ponikowski, P.; Voors, A.A.; Anker, S.D.; Bueno, H.; Cleland, J.G.F.; Coats, A.J.S.; Falk, V.; González-Juanatey, J.R.; Harjola, V.-P.; Jankowska, E.A.; et al. 2016 ESC Guidelines for the diagnosis and treatment of acute and chronic heart failure: The Task Force for the diagnosis and treatment of acute and chronic heart failure of the European Society of Cardiology (ESC)Developed with the special contribution of the Heart Failure Association (HFA) of the ESC. Eur. Heart J. 2016, 37, 2129-2200. [CrossRef]

26. McDonagh, T.A.; Metra, M.; Adamo, M.; Gardner, R.S.; Baumbach, A.; Böhm, M.; Burri, H.; Butler, J.; Čelutkienè, J.; Chioncel, O.; et al. 2021 ESC Guidelines for the diagnosis and treatment of acute and chronic heart failure. Eur. Heart J. 2021, 42, 3599-3726. [CrossRef]

27. Thygesen, K.; Alpert, J.S.; Jaffe, A.S.; Chaitman, B.R.; Bax, J.J.; Morrow, D.A.; White, H.D. Fourth Universal Definition of Myocardial Infarction (2018). J. Am. Coll. Cardiol. 2018, 72, 2231-2264. [CrossRef]

28. Rivera-Caravaca, J.M.; Núñez-Gil, I.J.; Vivas, D.; Viana-Llamas, M.C.; Uribarri, A.; Becerra-Muñoz, V.M.; Trabattoni, D.; Rozas, I.F.; Feltes, G.; López-Pais, J.; et al. Clinical profile and prognosis in patients on oral anticoagulation before admission for COVID-19. Eur. J. Clin. Investig. 2020, 51, e13436. [CrossRef]

29. Tremblay, D.; Van Gerwen, M.; Alsen, M.; Thibaud, S.; Kessler, A.J.; Venugopal, S.; Makki, I.; Qin, Q.; Dharmapuri, S.; Jun, T.; et al. Impact of anticoagulation prior to COVID-19 infection: A propensity score-matched cohort study. Blood 2020, 136, 144-147. [CrossRef]

30. Kornej, J.; Börschel, C.S.; Benjamin, E.J.; Schnabel, R.B. Epidemiology of Atrial Fibrillation in the 21st Century. Circ. Res. 2020, 127, 4-20. [CrossRef]

31. Bavishi, A.; Patel, R.B. Addressing Comorbidities in Heart Failure. Heart Fail. Clin. 2020, 16, 441-456. [CrossRef]

32. Tomasoni, D.; Italia, L.; Adamo, M.; Inciardi, R.M.; Lombardi, C.M.; Solomon, S.D.; Metra, M. COVID-19 and heart failure: From infection to inflammation and angiotensin II stimulation. Searching for evidence from a new disease. Eur. J. Heart Fail. 2020, 22, 957-966. [CrossRef]

33. Wu, C.; Chen, X.; Cai, Y.; Xia, J.; Zhou, X.; Xu, S.; Huang, H.; Zhang, L.; Zhou, X.; Du, C.; et al. Risk Factors Associated with Acute Respiratory Distress Syndrome and Death in Patients with Coronavirus Disease 2019 Pneumonia in Wuhan, China. JAMA Intern. Med. 2020, 180, 934-943. [CrossRef]

34. Sama, I.; Ravera, A.; Santema, B.T.; Van Goor, H.; Ter Maaten, J.M.; Cleland, J.G.F.; Rienstra, M.; Friedrich, A.W.; Samani, N.J.; $\mathrm{Ng}$, L.L.; et al. Circulating plasma concentrations of angiotensin-converting enzyme 2 in men and women with heart failure and effects of renin-angiotensin-aldosterone inhibitors. Eur. Heart J. 2020, 41, 1810-1817. [CrossRef]

35. Varga, Z.; Flammer, A.J.; Steiger, P.; Haberecker, M.; Andermatt, R.; Zinkernagel, A.S.; Mehra, M.R.; Schuepbach, R.A.; Ruschitzka, F.; Moch, H. Endothelial cell infection and endotheliitis in COVID-19. Lancet 2020, 395, 1417-1418. [CrossRef]

36. Schiavone, M.; Gasperetti, A.; Mancone, M.; Kaplan, A.V.; Gobbi, C.; Mascioli, G.; Busana, M.; Saguner, A.M.; Mitacchione, G.; Giacomelli, A.; et al. Redefining the Prognostic Value of High-Sensitivity Troponin in COVID-19 Patients: The Importance of Concomitant Coronary Artery Disease. J. Clin. Med. 2020, 9, 3263. [CrossRef] [PubMed]

37. Peterson, E.; Lo, K.B.; DeJoy, R.; Salacup, G.; Pelayo, J.; Bhargav, R.; Gul, F.; Albano, J.; Azmaiparashvili, Z.; Amanullah, A.; et al. The relationship between coronary artery disease and clinical outcomes in COVID-19. Coron. Artery Dis. 2020, 32, 367-371. [CrossRef]

38. Schiavone, M.; Gobbi, C.; Biondi-Zoccai, G.; D’Ascenzo, F.; Palazzuoli, A.; Gasperetti, A.; Mitacchione, G.; Viecca, M.; Galli, M.; Fedele, F.; et al. Acute Coronary Syndromes and Covid-19: Exploring the Uncertainties. J. Clin. Med. 2020, 9, 1683. [CrossRef]

39. Barman, H.A.; Atici, A.; Sahin, I.; Alici, G.; Tekin, E.A.; Baycan, F.; Ozturk, F.; Oflar, E.; Tugrul, S.; Yavuz, M.B.; et al. Prognostic significance of cardiac injury in COVID-19 patients with and without coronary artery disease. Coron. Artery Dis. 2020, 32, 359-366. [CrossRef]

40. Sokolski, M.; Zymlinski, R.; Biegus, J.; Siwołowski, P.; Nawrocka-Millward, S.; Todd, J.; Yerramilli, M.R.; Estis, J.; A Jankowska, E.; Banasiak, W.; et al. Urinary levels of novel kidney biomarkers and risk of true worsening renal function and mortality in patients with acute heart failure. Eur. J. Heart Fail. 2017, 19, 760-767. [CrossRef]

41. Alvarez-Garcia, J.; Lee, S.; Gupta, A.; Cagliostro, M.; Joshi, A.A.; Rivas-Lasarte, M.; Contreras, J.; Mitter, S.S.; LaRocca, G.; Tlachi, P.; et al. Prognostic Impact of Prior Heart Failure in Patients Hospitalized with COVID-19. J. Am. Coll. Cardiol. 2020, 76, 2334-2348. [CrossRef]

42. Rey, J.R.; Caro-Codón, J.; Rosillo, S.O.; Iniesta, Á.M.; Castrejón-Castrejón, S.; Marco-Clement, I.; Martín-Polo, L.; Merino-Argos, C.; Rodríguez-Sotelo, L.; García-Veas, J.M.; et al. Heart failure in COVID-19 patients: Prevalence, incidence and prognostic implications. Eur. J. Heart. Fail. 2020, 22, 2205-2215. [CrossRef]

43. Rumery, K.; Seo, A.; Jiang, L.; Choudhary, G.; Shah, N.R.; Rudolph, J.L.; Wu, W.; Erqou, S. Outcomes of coronavirus disease-2019 among veterans with pre-existing diagnosis of heart failure. ESC Heart Fail. 2021, 8, 2338-2344. [CrossRef] [PubMed]

44. Anyu, A.T.; Badawy, L.; Cannata, A.; Bromage, D.I.; Rind, I.A.; Albarjas, M.; Piper, S.; Shah, A.M.; McDonagh, T.A. Long-term outcomes after heart failure hospitalization during the COVID-19 pandemic: A multisite report from heart failure referral centers in London. ESC Heart Fail. 2021. [CrossRef] 